\title{
Serum ferritin levels are associated with insulin resistance in Chinese men and post-menopausal women: the Shanghai Changfeng study
}

\author{
Hui $\mathrm{Ma}^{1}$, Huandong $\mathrm{Lin}^{2}$, Yu Hu${ }^{1}$, Xiaoming $\mathrm{Li}^{2}$, Wanyuan $\mathrm{He}^{3}$, Xuejuan $\mathrm{Jin}^{4}$, Jian Gao ${ }^{5}$, Naiqing Zhao ${ }^{6}$, \\ Boshen $\mathrm{Pan}^{7}$ and Xin $\mathrm{GaO}^{2 *}$ \\ ${ }^{1}$ Department of Geriatrics, Zhongshan Hospital, Fudan University, Shanghai 200032, People's Republic of China \\ ${ }^{2}$ Department of Endocrinology and Metabolism, Zhongshan Hospital, Fudan University, Shanghai 200032, People's \\ Republic of China \\ ${ }^{3}$ Department of Ultrasonography, Zhongshan Hospital, Fudan University, Shanghai 200032, People's Republic of China \\ ${ }^{4}$ Clinical Epidemiology Center, Zhongshan Hospital, Fudan University, Shanghai 200032, People's Republic of China \\ ${ }^{5}$ Department of Clinical Nutrition, Zhongshan Hospital, Fudan University, Shanghai 200032, People's Republic of China \\ ${ }^{6}$ Department of Biostatistics, College of Public Health, Fudan University, Shanghai 200032, People's Republic of China \\ ${ }^{7}$ Department of Laboratory Medicine, Zhongshan Hospital, Fudan University, Shanghai 20032, People's Republic of China
}

(Submitted 25 April 2018 - Final revision received 12 June 2018 - Accepted 2 July 2018)

\begin{abstract}
Associations between ferritin and insulin sensitivity have been described in recent studies. The possible association showed conflicting results by sex and menopausal status. We aimed to investigate the cross-sectional association of ferritin levels with insulin resistance and $\beta$-cell function. A total of 2518 participants (1033 men, 235 pre-menopausal women and 1250 post-menopausal women) were enrolled from the Changfeng Study. A standard interview was conducted, as well as anthropometric measurements and laboratory analyses, for each participant. The serum ferritin level was measured using electrochemiluminescence immunoassay. Insulin resistance and $\beta$-cell function indices were derived from a homeostasis model assessment. The results showed that the serum ferritin levels were 250.4 (SD 165.2), 94.6 (SD 82.0) and 179.8 (SD 126.6$) \mathrm{ng} / \mathrm{ml}$ in the men, pre-menopausal and post-menopausal women, respectively. In fully adjusted models (adjusting for age, current smoking, BMI, waist:hip ratio, systolic blood pressure, diastolic blood pressure, TAG, HDL-cholesterol, LDL-cholesterol, log urine albumin: creatinine ratio, leucocytes, alanine aminotransferase, aspartate aminotransferase and $\gamma$-glutamyl transpeptidase), serum ferritin concentrations are significantly associated with insulin resistance in men and post-menopausal females, and the null association was observed in premenopausal females. Interestingly, an increased $\beta$-cell function associated with higher ferritin was observed in post-menopausal participants, but not in male participants. In conclusion, these results suggested that elevated serum ferritin levels were associated with surrogate measures of insulin resistance among the middle-aged and elderly male and post-menopausal women, but not in pre-menopausal women.
\end{abstract}

Key words: Ferritin: Insulin resistance: $\beta$-Cell function: Glucose homoeostasis

Serum ferritin is the widely used marker for total body Fe stores in epidemiological studies, which accurately reflects differences in body $\mathrm{Fe}$ stores by sex and age ${ }^{(1)}$. Accumulating studies have reported serum ferritin concentrations are associated with insulin resistance (IR) measured by homoeostasis model assessment (HOMA-IR) and abnormal pancreatic $\beta$-cell function $^{(2-4)}$. However, previous studies investigating this association have demonstrated conflicting results ${ }^{(2-5)}$. Bonfils et al. ${ }^{(6)}$ investigated 6392 individuals from the Danish general population and observed that elevated fasting serum ferritin levels were associated with surrogate measures of impaired $\beta$-cell function in both men and women, whereas the association with decreased insulin sensitivity was observed among men and older women but not among younger women. A crosssectional study of 12090 participants in Korea reported that increased serum ferritin concentrations were associated with IR in men, but not in women ${ }^{(7)}$, whereas the survey of 8235 participants in China reported that elevated serum ferritin levels were associated with HOMA-IR independent of several confounders in both men and women ${ }^{(8)}$. Similarly, another study conducted in China with 2786 people (both sexes) with no previous history of metabolic disorders found a positive association between serum ferritin levels and HOMA-IR values (adjusted for age, BMI, TAG and systolic blood pressure $(\mathrm{SBP}))^{(3)}$. In contrast, serum ferritin was not independently associated with IR as assessed using HOMA-IR in men or

Abbreviations: ALT, alanine transaminase; AST, aspartate transaminase; DM, diabetes mellitus; GGT, $\gamma$-glutamyl transpeptidase; HOMA-\%B, homeostatic model assessment for $\beta$-cell function; HOMA-IR, homeostasis model assessment index for insulin resistance; SBP, systolic blood pressure; UACR, urine albumin: creatinine ratio; WHR, waist:hip ratio.

* Corresponding author: X. Gao, fax +86 21 64037269, email zhongshan_endo@126.com 
women without diabetes in Western Australia( ${ }^{(5)}$. Therefore, the role played by ferritin in IR in men and women, especially among the Chinese population, remains controversial and poorly understood. Serum ferritin concentrations differ significantly according to sex and ethnicity ${ }^{(9)}$. In addition, there may be inadequate consideration of potential confounding effects on the relationship. Although these studies do not agree, we can still consider that the serum ferritin concentrations could reflect the development of glucose homoeostasis and that there are differences between the sexes. Further studies are needed to clarify the sex differences and menopausal status among the Chinese population.

Thus, we investigated the association between body Fe as assessed by the serum ferritin concentration and glucose homoeostasis using HOMA of IR and $\beta$-cell function in the community-based population of Chinese middle-aged and elderly men and women.

\section{Methods}

\section{Study population}

This cross-sectional study used data obtained from the Changfeng study, a community-based study of chronic diseases among the middle-aged and elderly, which has been described elsewhere ${ }^{(10)}$. From June 2009 to June 2012, 5375 participants were initially enrolled. Selection of the participants in the study was performed using the following exclusion criteria: lack of physical examination and laboratory assessments ( $n 54$ ), use of drugs known to influence Fe metabolism including the use of post-menopausal hormone therapy, mineral supplements (including Fe), vitamins and glucocorticoid ( $n$ 491), prevalent CVD (myocardial infarction, stroke or peripheral arterial disease) ( $n$ 379), prevalent haemodialysis ( $n$ 5), having liver disease (e.g. serum liver enzyme (aspartate aminotransferase (AST) and alanine aminotransferase (ALT)) activities above two times of the upper normal limits), increased serum creatinine $(>124 \mu \mathrm{mol} / \mathrm{l})$, tumour, chronic inflammatory disease (e.g. rheumatoid arthritis, Crohn's disease) and/or a disorder of Fe metabolism (e.g. aplastic anaemia, $\beta$-thalassemia major and haemochromatosis) ( $n$ 403), diagnosed diabetes mellitus (DM) or the use of hypoglycaemic medications ( $n$ 366), diagnosed hypertension or the use of antihypertensive medications ( $n$ 1009), participants with laboratory evidences of inflammation or anaemia (leucocyte count $>10000 / \mu \mathrm{l}$, platelet count $>300000 / \mu \mathrm{l}$, haemoglobin $<11 \mathrm{~g} / \mathrm{l}$ ) ( $n$ 149), having received a blood transfusion or donated blood in the past 3 months ( $n$ 1). Finally, 2518 participants were included in the analysis.

The study protocol, consent form, and participant-related materials were approved by the ethical committee of Zhongshan Hospital, Fudan University and all procedures were performed in accordance with the principles of the Declaration of Helsinki and later amendments. Informed consents were obtained from all individuals taking part in the current investigation. Interviews and physical examinations were performed at the Changfeng Community Health Service Center.

\section{Clinical measurements}

Letters were sent to participants with instructions asking them not to alter their diet or level of physical activity for at least $3 \mathrm{~d}$ before the test. A questionnaire was administered by trained nurses to evaluate the medical history and lifestyle of each participant. All measurements and the sample collections were performed in the morning after overnight fasting. Body weight and height were assessed for the determination of BMI, which was calculated as the weight divided by the height squared $\left(\mathrm{kg} / \mathrm{m}^{2}\right.$ ). The waist:hip ratio (WHR) was calculated as the waist circumference divided by the hip circumference. The resting blood pressure was measured three times, and the mean value was used for the analysis. Serum was examined for glucose, total cholesterol (TC), HDL-cholesterol, TAG and liver enzymes using a model 7600 automated bio-analyser (Hitachi). The level of LDL-cholesterol was calculated using the Friedewald equation. The fasting blood glucose (FBG) and $2 \mathrm{~h}$ glucose levels following a 75-g oral glucose challenge (oral glucose tolerance test (OGTT) $2 \mathrm{~h}$ blood glucose (PPG)) for non-diabetics were measured using the glucose oxidase method. The serum samples, which had been stored at $-80^{\circ} \mathrm{C}$ since the time of the baseline survey, were used for serum ferritin and insulin level measurements. Serum ferritin and insulin were measured using electrochemiluminescence immunoassay using an immunoassay analyzer (Cobas-6001; Roche; CV $<4.0$ and $<5.0 \%$, respectively). The values of HOMA-IR index widely used for assessment of IR were calculated using the standard formula: $\mathrm{HOMA}-\mathrm{IR}=($ glucose $\times$ insulin $) / 22 \cdot 5$. The values of homeostatic model assessment $\beta$-cell function (HOMA- $\% \mathrm{~B}$ ) were calculated using the formula: $\mathrm{HOMA}-\% \mathrm{~B}=(20 \times$ insulin $) /$ $(\text { glucose }-23 \cdot 5)^{(11)}$.

Hypertension was based on the Seventh Report of the Joint National Committee ${ }^{(12)}$. The diagnoses of impaired fasting glucose, impaired glucose tolerance and DM were determined using the American Diabetes Association 2010 criteria $^{(13)}$. The diagnosis of CVD was according to self-reporting and confirmed using medical records.

\section{Statistical analysis}

The data were analysed using SPSS 16.0 for Windows (SPSS Inc.). The participants were categorised into serum ferritin tertiles as follows: (Q1, 8.1-164.0; Q2, 164.1-274.0; Q3, $274 \cdot 1-1160 \cdot 0 \mathrm{ng} / \mathrm{ml}$ in males; Q1, 5·5-49.6; Q2, 51·0-98.7; Q3, $102 \cdot 3-476 \cdot 0 \mathrm{ng} / \mathrm{ml}$ in pre-menopausal females; Q1, 8.2-120.0; Q2, 120.4-196.8; Q3, 197.5-1488.0 ng/ml in post-menopausal females). Skewed variables were logarithmically transformed to improve normality before analysis. The results were expressed as means and standard deviations or percentage and standard errors for quantitative variables. A weighted one-way ANOVA test or $\chi^{2}$ test was used to compare the means of different variables between three categories in the field. Analysis of covariance and logistic regression, with adjustments for the potential confounding factors, were conducted to compare means and proportions, respectively, across the ferritin tertiles. Two-sided values of $P$ value $<0.05$ were considered statistically significant. 


\section{Results}

\section{Population characteristics}

A total of 1033 males and 1485 females were evaluated. The characteristics of the study participants are shown in Table 1. The mean value of ferritin was $200 \cdot 8$ (sD 148.5) ng/ml. The mean concentrations of serum ferritin were significantly lower in premenopausal women compared with post-menopausal women (94.6 (sD 82.0) v. 179.8 ( $\mathrm{sD} 126 \cdot 6) \mathrm{ng} / \mathrm{ml}$, respectively) and still lower in post-menopausal women compared with men (179.8 (SD 126.6) v. 250.4 (SD 165.2) $\mathrm{ng} / \mathrm{ml}$, respectively). A total of $22.2 \%$ of the participants were current smokers. The differences among men, pre-menopausal women and post-menopausal women in terms of age, BMI, WHR, SBP, diastolic blood pressure (DBP), ALT, AST, $\gamma$-glutamyl transpeptidase (GGT), leucocytes, TC, LDL-cholesterol, HDL-cholesterol, TAG, FBG, PPG, HOMAIR, HOMA-\%B, urine albumin:creatinine ratio (UACR) and the presence of current smokers were statistically significant.

\section{Characteristics of the participants according to the ferritin tertiles}

Participant characteristics across the tertiles of serum ferritin concentrations in men and women are summarised in Table 2.
In males, when the traditional risk factors were examined, age, BMI, WHR, SBP, DBP, ALT, AST, GGT, leucocytes, TC, LDLcholesterol, HDL-cholesterol, TAG, FBG, PPG, HOMA-IR, HOMA- $\% \mathrm{~B}$ and UACR were significantly associated with the ferritin tertiles. In pre-menopausal females, GGT, LDL-cholesterol, HDL-cholesterol and TAG were significantly associated with the tertiles groups. In post-menopausal females, age, BMI, WHR, ALT, AST, GGT, TC, LDL-cholesterol, HDL-cholesterol, TAG, FBG, PPG, HOMA-IR and HOMA-\%B were significantly associated with the ferritin tertiles. Other parameters were not significantly different among the tertile groups.

\section{Association between ferritin and glucose homoeostasis}

Table 3 presents the IR measured by HOMA-IR and HOMA-\%B of the participants according to the ferritin tertiles and sexes. Higher concentrations of serum ferritin were significantly associated with higher values for IR after adjusting for age, current smoking, BMI, WHR, SBP, DBP, TAG, HDL-cholesterol, LDLcholesterol, logUACR and leucocytes (model 2) in men. Further adjustment for ALT, AST and GGT (model 3) did not materially attenuate the association of serum ferritin with HOMA-IR $\left(P_{\text {for trend }}=0.001\right)$, although the association with HOMA-\%B remained statistically non-significant $\left(P_{\text {for trend }}=0 \cdot 432\right)$. In fully

Table 1. Characteristics of the study participants

(Mean values and standard deviations; numbers and percentages; medians and interquartile ranges (IQR))

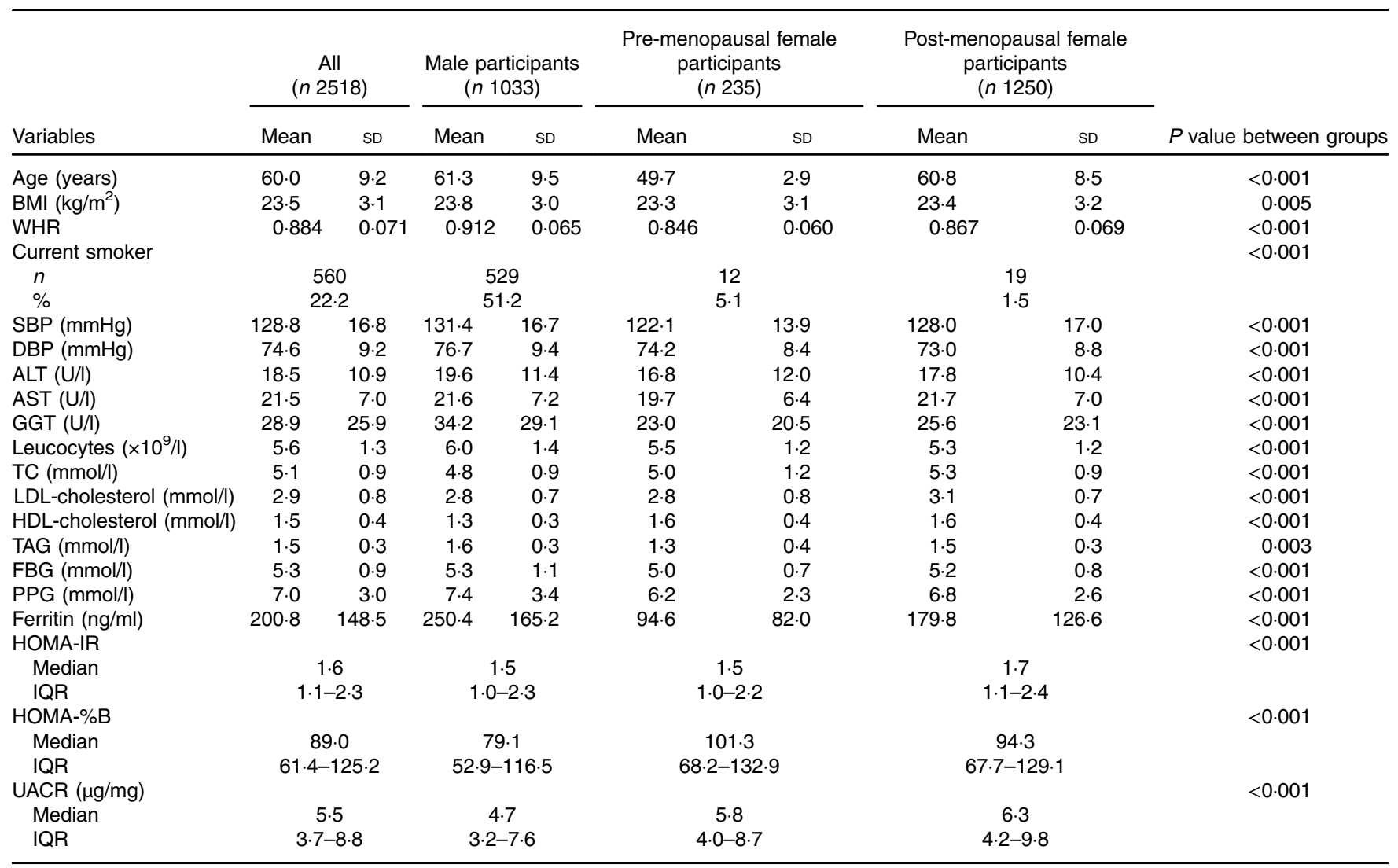

WHR, waist:hip ratio; SBP, systolic blood pressure; DBP, diastolic blood pressure; ALT, alanine aminotransferase; AST, aspartate aminotransferase; GGT, $\gamma$-glutamyl transpeptidase; TC, total cholesterol; FBG, fasting blood glucose; PPG, oral glucose tolerance test $2 \mathrm{~h}$ blood glucose; HOMA-IR, homoeostasis model assessment index for insulin resistance; HOMA-\%B, homoeostasis model assessment index for $\beta$-cell function; UACR, urine albumin:creatinine ratio. 
Table 2. Characteristics of the participants according to tertile groups for ferritin in male, pre-menopausal and post-menopausal female participants (Mean values and standard deviations; numbers and percentages; medians and interquartile ranges (IQR))

\begin{tabular}{|c|c|c|c|c|c|c|c|}
\hline \multirow[b]{3}{*}{ Variables } & \multicolumn{6}{|c|}{ Tertile groups for ferritin in male participants } & \multirow[b]{3}{*}{$P$ among groups } \\
\hline & \multicolumn{2}{|c|}{ First tertile (n 344) } & \multicolumn{2}{|c|}{ Second tertile (n 343) } & \multicolumn{2}{|c|}{ Third tertile ( $n$ 346) } & \\
\hline & Mean & $\mathrm{SD}$ & Mean & SD & Mean & $\mathrm{SD}$ & \\
\hline Ferritin range (ng/ml) & \multicolumn{2}{|c|}{$8 \cdot 1-164 \cdot 0$} & \multicolumn{2}{|c|}{$164 \cdot 1-274 \cdot 0$} & \multicolumn{2}{|c|}{$274 \cdot 1-1160 \cdot 0$} & \\
\hline Age (years) & $62 \cdot 5$ & $9 \cdot 7$ & $60 \cdot 8^{\star}$ & $9 \cdot 0$ & $60 \cdot 6^{*}$ & $9 \cdot 6$ & 0.011 \\
\hline BMI $\left(\mathrm{kg} / \mathrm{m}^{2}\right)$ & $23 \cdot 1$ & $3 \cdot 1$ & $23 \cdot 8^{*}$ & 2.9 & $24.5^{\star} \dagger$ & 2.9 & $<0.001$ \\
\hline WHR & 0.900 & 0.068 & $0.912^{*}$ & 0.067 & $0.924^{*} \dagger$ & 0.056 & $<0.001$ \\
\hline Current smoker & & & & & & & 0.932 \\
\hline$n$ & \multicolumn{2}{|c|}{165} & \multicolumn{2}{|c|}{169} & \multicolumn{2}{|c|}{170} & \\
\hline$\%$ & \multicolumn{2}{|c|}{$32 \cdot 7$} & \multicolumn{2}{|c|}{33.5} & \multicolumn{2}{|c|}{33.7} & \\
\hline $\mathrm{SBP}(\mathrm{mmHg})$ & $130 \cdot 3$ & $17 \cdot 5$ & $130 \cdot 5$ & $16 \cdot 3$ & $133 \cdot 3^{\star} \dagger$ & $16 \cdot 3$ & 0.036 \\
\hline $\mathrm{DBP}(\mathrm{mmHg})$ & $75 \cdot 2$ & 9.4 & 76.4 & $9 \cdot 0$ & $78 \cdot 7^{\star} \dagger$ & $9 \cdot 4$ & $<0.001$ \\
\hline ALT $(\mathrm{U} / \mathrm{l})$ & $16 \cdot 0$ & $8 \cdot 1$ & $18 \cdot 9^{*}$ & 9.9 & $23 \cdot 7^{\star} \dagger$ & 13.9 & $<0.001$ \\
\hline AST (U/I) & $20 \cdot 8$ & $7 \cdot 4$ & 21.0 & 5.5 & $23 \cdot 0^{*} \dagger$ & $8 \cdot 1$ & $<0.001$ \\
\hline GGT (U/I) & $28 \cdot 4$ & $25 \cdot 0$ & $32 \cdot 4$ & $22 \cdot 6$ & $41 \cdot 7^{*} \dagger$ & $36 \cdot 3$ & $<0.001$ \\
\hline Leucocytes $\left(\times 10^{9} / I\right)$ & $5 \cdot 8$ & $1 \cdot 3$ & $6 \cdot 1^{*}$ & 1.4 & $6 \cdot 1^{*}$ & 1.4 & 0.008 \\
\hline $\mathrm{TC}(\mathrm{mmol} / \mathrm{l})$ & $4 \cdot 6$ & 0.8 & $4 \cdot 8^{*}$ & 0.9 & $4 \cdot 9^{\star} \dagger$ & 0.8 & $<0.001$ \\
\hline LDL-cholesterol (mmol/l) & $2 \cdot 7$ & 0.7 & $2 \cdot 8$ & 0.7 & $2 \cdot 8^{*}$ & 0.8 & 0.006 \\
\hline HDL-cholesterol (mmol/l) & 1.4 & 0.4 & $1 \cdot 3^{*}$ & 0.4 & $1 \cdot 3^{*}$ & 0.3 & 0.001 \\
\hline TAG $(\mathrm{mmol} / \mathrm{l})$ & $1 \cdot 3$ & 0.7 & $1 \cdot 6^{*}$ & $1 \cdot 3$ & $1 \cdot 8^{*} \dagger$ & $1 \cdot 1$ & $<0.001$ \\
\hline FBG (mmol/l) & $5 \cdot 1$ & 0.6 & $5 \cdot 3^{*}$ & 0.9 & $5 \cdot 6^{\star} \dagger$ & $1 \cdot 1$ & $<0.001$ \\
\hline PPG (mmol/l) & $6 \cdot 5$ & $2 \cdot 5$ & $7 \cdot 2^{*}$ & $3 \cdot 1$ & $8 \cdot 4^{*} \dagger$ & $4 \cdot 2$ & $<0.001$ \\
\hline HOMA-IR & & & & & & & $<0.001$ \\
\hline Median & \multicolumn{2}{|c|}{$1 \cdot 2$} & \multicolumn{2}{|c|}{$1.5^{\star}$} & \multicolumn{2}{|c|}{$1.9^{*} \dagger$} & \\
\hline IQR & \multicolumn{2}{|c|}{$0 \cdot 7-1 \cdot 8$} & \multicolumn{2}{|c|}{$1 \cdot 0-2 \cdot 2$} & \multicolumn{2}{|c|}{$1 \cdot 2-2 \cdot 8$} & \\
\hline HOMA-\%B & & & & & & & 0.012 \\
\hline Median & \multicolumn{2}{|c|}{74.5} & \multicolumn{2}{|c|}{$79 \cdot 1$} & \multicolumn{2}{|c|}{$85 \cdot 8^{*}$} & \\
\hline IQR & \multicolumn{2}{|c|}{$48 \cdot 0-104 \cdot 3$} & \multicolumn{2}{|c|}{$53 \cdot 3-112 \cdot 8$} & \multicolumn{2}{|c|}{$55 \cdot 2-130 \cdot 3$} & \\
\hline UACR $(\mu \mathrm{g} / \mathrm{mg})$ & & & & & & & 0.015 \\
\hline Median & \multicolumn{2}{|c|}{4.4} & \multicolumn{2}{|c|}{4.6} & \multicolumn{2}{|c|}{$5 \cdot 2^{\star} \dagger$} & \\
\hline IQR & & & & & & & \\
\hline
\end{tabular}

Tertile groups for ferritin in pre-menopausal female participants

\begin{tabular}{|c|c|c|c|c|c|c|c|}
\hline \multirow[b]{2}{*}{ Variables } & \multicolumn{2}{|c|}{ First tertile $(n 78)$} & \multicolumn{2}{|c|}{ Second tertile $(n 78)$} & \multicolumn{2}{|c|}{ Third tertile $(n 79)$} & \multirow[b]{2}{*}{$P$ among groups } \\
\hline & Mean & SD & Mean & SD & Mean & SD & \\
\hline Ferritin range $(\mathrm{ng} / \mathrm{ml})$ & \multicolumn{2}{|c|}{$5.5-49.6$} & \multicolumn{2}{|c|}{$51 \cdot 0-98 \cdot 7$} & \multicolumn{2}{|c|}{$102 \cdot 3-476 \cdot 0$} & \\
\hline Age (years) & $49 \cdot 2$ & $2 \cdot 2$ & 49.9 & 2.6 & 49.9 & 3.8 & 0.219 \\
\hline BMI $\left(\mathrm{kg} / \mathrm{m}^{2}\right)$ & $22 \cdot 8$ & 3.0 & $23 \cdot 3$ & $2 \cdot 9$ & $23.8^{*}$ & 3.2 & 0.135 \\
\hline WHR & 0.843 & 0.072 & 0.841 & 0.052 & 0.855 & 0.054 & 0.289 \\
\hline Current smoker & & & & & & & 0.347 \\
\hline$n$ & \multicolumn{2}{|c|}{6} & \multicolumn{2}{|c|}{2} & \multicolumn{2}{|c|}{4} & \\
\hline$\%$ & \multicolumn{2}{|c|}{$7 \cdot 7$} & \multicolumn{2}{|c|}{$2 \cdot 6$} & \multicolumn{2}{|c|}{$5 \cdot 1$} & \\
\hline $\mathrm{SBP}(\mathrm{mmHg})$ & $123 \cdot 2$ & 14.5 & $120 \cdot 7$ & 14.3 & $122 \cdot 6$ & $12 \cdot 9$ & 0.498 \\
\hline $\mathrm{DBP}(\mathrm{mmHg})$ & $74 . \overline{5}$ & 8.9 & 72.9 & $8 \cdot 1$ & $75 \cdot 2$ & $8 \cdot 1$ & 0.214 \\
\hline ALT (U/l) & $15 \cdot 4$ & 9.8 & $15 \cdot 9$ & $10 \cdot 4$ & $19 \cdot 1$ & $13 \cdot 1$ & 0.120 \\
\hline AST (U/I) & 19.4 & 6.5 & $19 \cdot 4$ & $5 \cdot 2$ & 20.5 & $7 \cdot 3$ & 0.439 \\
\hline GGT (U/I) & $21 \cdot 2$ & $20 \cdot 0$ & $21 \cdot 1$ & $12 \cdot 0$ & $27 \cdot 6^{*} \dagger$ & $25 \cdot 6$ & 0.045 \\
\hline Leucocytes $\left(\times 10^{9} / \mathrm{I}\right)$ & 5.7 & 1.2 & $5 \cdot 3$ & 1.2 & 5.5 & 1.3 & $0 \cdot 169$ \\
\hline TC $(\mathrm{mmol} / \mathrm{l})$ & 4.8 & 0.9 & $5 \cdot 1^{*}$ & 0.9 & 5.0 & 0.7 & 0.112 \\
\hline LDL-cholesterol (mmol/l) & $2 \cdot 7$ & 0.9 & $3.0^{*}$ & 0.8 & 2.8 & 0.7 & 0.048 \\
\hline HDL-cholesterol (mmol/l) & 1.6 & 0.4 & 1.6 & 0.4 & $1.5 t$ & 0.3 & 0.036 \\
\hline TAG (mmol/l) & 1.3 & 0.6 & 1.2 & 0.5 & $1.5^{\star} \dagger$ & 0.8 & 0.003 \\
\hline $\mathrm{FBG}(\mathrm{mmol} / \mathrm{L})$ & $5 \cdot 0$ & 0.4 & 4.9 & 0.5 & $5 \cdot 2^{*} \dagger$ & 1.0 & 0.050 \\
\hline $\mathrm{PPG}(\mathrm{mmol} / \mathrm{l})$ & 5.9 & 1.7 & $6 \cdot 1$ & 1.7 & $6 \cdot 7^{*}$ & $3 \cdot 1$ & 0.100 \\
\hline HOMA-IR & & & & & & & 0.058 \\
\hline Median & \multicolumn{2}{|c|}{1.4} & \multicolumn{2}{|c|}{1.5} & \multicolumn{2}{|c|}{$1.6 \dagger$} & \\
\hline IQR & \multicolumn{2}{|c|}{$1 \cdot 0-2 \cdot 0$} & \multicolumn{2}{|c|}{$0.9-2 \cdot 0$} & \multicolumn{2}{|c|}{$1 \cdot 1-2 \cdot 5$} & \\
\hline HOMA-\%B & \multirow{2}{*}{\multicolumn{2}{|c|}{$100 \cdot 0$}} & & & & & 0.827 \\
\hline Median & & & \multicolumn{2}{|c|}{98.7} & \multicolumn{2}{|c|}{$105 \cdot 3$} & \\
\hline IQR & \multicolumn{2}{|c|}{$65 \cdot 3-128 \cdot 7$} & \multicolumn{2}{|c|}{$65 \cdot 7-131 \cdot 4$} & \multicolumn{2}{|c|}{$68 \cdot 4-136 \cdot 0$} & \\
\hline UACR $(\mu \mathrm{g} / \mathrm{mg})$ & & & & & & & 0.719 \\
\hline Median & \multirow{2}{*}{\multicolumn{2}{|c|}{$\begin{array}{c}6 \cdot 1 \\
4 \cdot 0-8 \cdot 3\end{array}$}} & \multirow{2}{*}{\multicolumn{2}{|c|}{$\begin{array}{c}5 \cdot 3 \\
4 \cdot 0-9 \cdot 1\end{array}$}} & \multirow{2}{*}{\multicolumn{2}{|c|}{$\begin{array}{c}5 \cdot 9 \\
4 \cdot 0-8 \cdot 6\end{array}$}} & \\
\hline IQR & & & & & & & \\
\hline
\end{tabular}


Tertile groups for ferritin in post-menopausal female participants

\begin{tabular}{|c|c|c|c|c|c|c|c|}
\hline \multirow[b]{2}{*}{ Variables } & \multicolumn{2}{|c|}{ First tertile ( $n$ 417) } & \multicolumn{2}{|c|}{ Second tertile (n 417) } & \multicolumn{2}{|c|}{ Third tertile (n 416) } & \multirow[b]{2}{*}{$P$ among groups } \\
\hline & Mean & SD & Mean & SD & Mean & SD & \\
\hline Ferritin range (ng/ml) & \multicolumn{2}{|c|}{$8 \cdot 2-120 \cdot 0$} & \multicolumn{2}{|c|}{$120 \cdot 4-196 \cdot 8$} & \multicolumn{2}{|c|}{$197 \cdot 5-1488.0$} & \\
\hline Age (years) & $59 \cdot 1$ & 8.5 & $60 \cdot 6^{\star}$ & 8.4 & $62 \cdot 8^{\star} \dagger$ & $8 \cdot 3$ & $<0.001$ \\
\hline BMl $\left(\mathrm{kg} / \mathrm{m}^{2}\right)$ & 22.9 & $3 \cdot 1$ & $23 \cdot 3$ & $3 \cdot 2$ & $24 \cdot 1^{*} \dagger$ & $3 \cdot 0$ & $<0.001$ \\
\hline WHR & 0.854 & 0.063 & $0.865^{\star}$ & 0.068 & $0.883^{\star} \dagger$ & 0.073 & $<0.001$ \\
\hline Current smoker & & & & & & & 0.806 \\
\hline$n$ & \multicolumn{2}{|c|}{5} & \multicolumn{2}{|c|}{7} & \multicolumn{2}{|c|}{7} & \\
\hline$\%$ & \multicolumn{2}{|c|}{$1 \cdot 2$} & \multicolumn{2}{|c|}{$1 \cdot 7$} & \multicolumn{2}{|c|}{1.7} & \\
\hline $\mathrm{SBP}(\mathrm{mmHg})$ & $127 \cdot 2$ & $17 \cdot 0$ & $127 \cdot 2$ & $15 \cdot 9$ & $129 \cdot 6^{\star} \dagger$ & $17 \cdot 9$ & 0.055 \\
\hline $\mathrm{DBP}(\mathrm{mmHg})$ & 73.0 & 8.8 & 73.0 & 8.9 & 72.9 & $8 \cdot 6$ & 0.987 \\
\hline ALT (U/I) & $16 \cdot 4$ & $8 \cdot 4$ & $17 \cdot 0$ & $9 \cdot 3$ & $20 \cdot 1$ & 12.5 & $<0.001$ \\
\hline AST (U/I) & $21 \cdot 0$ & $6 \cdot 2$ & $21 \cdot 2$ & $6 \cdot 2$ & $22 \cdot 8$ & $8 \cdot 2$ & $<0.001$ \\
\hline GGT (U/I) & $22 \cdot 8$ & $17 \cdot 4$ & $25 \cdot 6$ & $20 \cdot 4$ & $28 \cdot 5^{\star}$ & 21.9 & 0.002 \\
\hline Leucocytes $\left(\times 10^{9} / \mathrm{I}\right)$ & $5 \cdot 2$ & $1 \cdot 1$ & $5 \cdot 3$ & $1 \cdot 2$ & 5.4 & $1 \cdot 3$ & 0.189 \\
\hline $\mathrm{TC}(\mathrm{mmol} / \mathrm{l})$ & $5 \cdot 2$ & 0.8 & $5 \cdot 3$ & 0.8 & 5.4 & 0.9 & $<0.001$ \\
\hline LDL-cholesterol (mmol/l) & 2.9 & 0.7 & $3 \cdot 1$ & 0.7 & $3 \cdot 1$ & 0.8 & 0.001 \\
\hline HDL-cholesterol (mmol/l) & $1 \cdot 6$ & 0.4 & 1.6 & 0.4 & 1.5 & 0.4 & $<0.001$ \\
\hline $\mathrm{TAG}(\mathrm{mmol} / \mathrm{l})$ & $1 \cdot 3$ & 0.6 & 1.5 & 0.8 & $1 \cdot 8$ & 1.4 & $<0.001$ \\
\hline FBG (mmol/l) & $5 \cdot 1$ & 0.8 & $5 \cdot 2$ & 0.7 & $5 \cdot 3$ & 0.9 & 0.002 \\
\hline PPG (mmol/l) & $6 \cdot 3$ & $2 \cdot 2$ & $6 \cdot 7$ & $2 \cdot 4$ & $7 \cdot 5$ & $3 \cdot 1$ & $<0.001$ \\
\hline \multicolumn{7}{|l|}{ HOMA-IR } & $<0.001$ \\
\hline Median & \multicolumn{2}{|c|}{1.4} & \multicolumn{2}{|c|}{$1 \cdot 6^{\star}$} & \multicolumn{2}{|c|}{$1.9^{*} \dagger$} & \\
\hline IQR & \multicolumn{2}{|c|}{$1 \cdot 0-2 \cdot 0$} & \multicolumn{2}{|c|}{$1 \cdot 1-2 \cdot 4$} & \multicolumn{2}{|c|}{$1 \cdot 4-2 \cdot 8$} & \\
\hline HOMA-\%B & & & & & & & $<0.001$ \\
\hline Median & \multicolumn{2}{|c|}{$85 \cdot 1$} & \multicolumn{2}{|c|}{$93 \cdot 3^{*}$} & \multicolumn{2}{|c|}{$103 \cdot 9^{*} \dagger$} & \\
\hline IQR & \multicolumn{2}{|c|}{$62 \cdot 9-113 \cdot 6$} & \multicolumn{2}{|c|}{$67 \cdot 1-132 \cdot 5$} & \multicolumn{2}{|c|}{$73 \cdot 1-136 \cdot 8$} & \\
\hline UACR $(\mu \mathrm{g} / \mathrm{mg})$ & & & & & & & 0.508 \\
\hline Median & \multirow{2}{*}{\multicolumn{2}{|c|}{$\begin{array}{c}6 \cdot 2 \\
4 \cdot 1-9 \cdot 2\end{array}$}} & \multirow{2}{*}{\multicolumn{2}{|c|}{$\begin{array}{c}6 \cdot 3 \\
4.3-9 \cdot 9\end{array}$}} & \multirow{2}{*}{\multicolumn{2}{|c|}{$\begin{array}{c}6 \cdot 5 \\
4 \cdot 2-10 \cdot 1\end{array}$}} & \\
\hline IQR & & & & & & & \\
\hline
\end{tabular}

WHR, waist:hip ratio; SBP, systolic blood pressure; DBP, diastolic blood pressure; ALT, alanine aminotransferase; AST, aspartate aminotransferase; GGT, $\gamma$-glutamyl transpeptidase; TC, total cholesterol; FBG, fasting blood glucose; PPG, oral glucose tolerance test $2 \mathrm{~h}$ blood glucose; HOMA-IR, homoeostasis model assessment index for insulin resistance; HOMA-\%B, homoeostasis model assessment index for $\beta$-cell function; UACR, urine albumin:creatinine ratio; LSD, least significant difference.

* ANOVA with LSD post hoc test or $X^{2}$ statistical analysis: $P<0.05 \mathrm{v}$. first tertile.

$\dagger$ ANOVA with LSD post hoc test or $X^{2}$ statistical analysis: $P<0.05 \mathrm{v}$. second tertile.

adjusted models (model 3), serum ferritin concentrations were significantly associated with IR and $\beta$-cell function in postmenopausal females, and the null association in premenopausal females remained in all models.

\section{Discussion}

In the present cross-sectional study, we found that a higher serum ferritin concentration was significantly associated with IR in male and post-menopausal participants, independently of possible risk factors. Furthermore, an increased $\beta$-cell function associated with higher ferritin level was observed in postmenopausal participants, but not in male participants.

Recent studies showed conflicting results with positive, negative or no associations between ferritin and IR in various populations with and without comorbidities ${ }^{(2-5)}$. We identified differences by sex and menopausal status in the relationship of ferritin with IR as significant associations were found in men and post-menopausal women, but not in pre-menopausal women, in our study. Our findings correspond well with the previous study by Lee et al. ${ }^{(14)}$. Lee et al conducted a crosssectional study of 6311 Korean adults older than 20 years who participated in the 2008 Korean National Health and Nutrition Examination Survey and found an association between serum ferritin and HOMA-IR in men and post-menopausal women but not in pre-menopausal women. Consistent with our findings, the previous studies ${ }^{(15)}$ showed no association between circulating ferritin concentrations and HOMA-IR in pre-menopausal women. The mean concentrations of serum ferritin in the premenopausal women in our study were nearly 2.5-fold lower than those in men, and the ferritin levels were about 2-fold lower in pre-menopausal women than those in postmenopausal women. This is likely attributable to menstrual Fe loss in pre-menopausal women. As serum ferritin differs significantly between men and women, it is thought that ferritin plays a different role in IR in each sex ${ }^{(16)}$. Theoretically, in men and post-menopausal women, the relationship between ferritin and IR might be more obvious because of higher Fe accumulation than that in pre-menopausal women. Moreover, changes in oestrogen levels may influence the association. The premenopausal women have a more insulin-sensitive environment than post-menopausal women due to different oestrogen levels $^{(17)}$, which may contribute to the different associations with the ferritin levels in pre-menopausal and post-menopausal women. The reduction in oestrogen levels seen at menopause has also been shown to increase levels of oxidative stress ${ }^{(18)}$, probably reinforcing the association between ferritin and IR. It has been reported that serum ferritin levels were lower in postmenopausal women using hormone therapy compared with 
Table 3. Homoeostasis model assessment index for insulin resistance (HOMA-IR) and homoeostasis model assessment index for $\beta$-cell function (HOMA-\%B) in the participants according to tertile groups for ferritin in male, pre-menopausal and post-menopausal female participants

(Mean values with their standard errors)

\begin{tabular}{|c|c|c|c|c|c|c|c|}
\hline & \multicolumn{7}{|c|}{ Tertile groups for ferritin in male participants } \\
\hline & \multicolumn{2}{|c|}{ First tertile } & \multicolumn{2}{|c|}{ Second tertile } & \multicolumn{2}{|c|}{ Third tertile } & \multirow[b]{2}{*}{$P$ value for trend } \\
\hline & Mean & SE & Mean & SE & Mean & SE & \\
\hline \multicolumn{8}{|l|}{$\log \mathrm{HOMA}-\mathrm{IR}$} \\
\hline Model $1^{*}$ & 0.075 & 0.015 & 0.185 & 0.015 & 0.269 & 0.015 & $<0.001$ \\
\hline Model $2 \dagger$ & 0.127 & 0.012 & $0.183 \ddagger$ & 0.011 & $0.217 \neq \S$ & 0.012 & $<0.001$ \\
\hline Model 3॥ & 0.141 & 0.011 & $0 \cdot 186 \ddagger$ & 0.011 & $0.200 \ddagger$ & 0.011 & 0.001 \\
\hline \multicolumn{8}{|l|}{ logHOMA-\%B } \\
\hline Model $1^{*}$ & 1.867 & 0.014 & 1.896 & 0.014 & $1.919 \ddagger$ & 0.014 & 0.035 \\
\hline Model $2 \dagger$ & 1.897 & 0.013 & 1.893 & 0.013 & 1.892 & 0.013 & 0.951 \\
\hline \multirow[t]{2}{*}{ Model 3\| } & 1.906 & 0.013 & 1.895 & 0.013 & $1 \cdot 881$ & 0.013 & 0.432 \\
\hline & \multicolumn{7}{|c|}{ Tertile groups for ferritin in pre-menopausal participants } \\
\hline \multicolumn{8}{|l|}{ logHOMA-IR } \\
\hline Model $1^{*}$ & 0.167 & 0.028 & 0.154 & 0.028 & $0.240 \S$ & 0.028 & 0.062 \\
\hline Model $2 \dagger$ & 0.174 & 0.024 & 0.185 & 0.024 & 0.203 & 0.024 & 0.696 \\
\hline Model 3॥ & 0.182 & 0.023 & 0.185 & 0.023 & 0.195 & 0.023 & 0.929 \\
\hline \multicolumn{8}{|l|}{ logHOMA-\%B } \\
\hline Model $1^{*}$ & 1.981 & 0.025 & 1.991 & 0.025 & 2.003 & 0.024 & 0.830 \\
\hline Model $2 \dagger$ & 1.982 & 0.023 & 2.015 & 0.023 & 1.977 & 0.023 & 0.452 \\
\hline \multirow[t]{2}{*}{ Model 3\| } & 1.980 & 0.023 & $2 \cdot 013$ & 0.023 & 1.982 & 0.023 & 0.512 \\
\hline & \multicolumn{7}{|c|}{ Tertile groups for ferritin in post-menopausal participants } \\
\hline \multicolumn{8}{|l|}{ logHOMA-IR } \\
\hline Model $1^{*}$ & 0.153 & 0.012 & $0.223 \ddagger$ & 0.012 & $0.308 \neq \S$ & 0.012 & $<0.001$ \\
\hline Model 2† & 0.180 & 0.011 & $0.231 \ddagger$ & 0.010 & $0.275 \neq \S$ & 0.011 & $<0.001$ \\
\hline Model 3\| & 0.186 & 0.010 & $0.233 \ddagger$ & 0.010 & $0.266 \neq \S$ & 0.011 & $<0.001$ \\
\hline \multicolumn{8}{|c|}{$\log \mathrm{HOMA}-\% \mathrm{~B}$} \\
\hline Model $1^{*}$ & 1.920 & 0.011 & $1.966 \ddagger$ & 0.011 & $2 \cdot 016 \neq \S$ & 0.011 & $<0.001$ \\
\hline Model $2 \nmid$ & 1.940 & 0.011 & $1.970 \ddagger$ & 0.010 & $1.993 \ddagger$ & 0.011 & 0.002 \\
\hline Model 3II & 1.942 & 0.011 & 1.971 & 0.010 & $1.990 \ddagger$ & 0.011 & 0.009 \\
\hline
\end{tabular}

WHR, waist:hip ratio; SBP, systolic blood pressure; DBP, diastolic blood pressure; UACR, urine albumin:creatinine ratio; ALT, alanine aminotransferase; AST, aspartate aminotransferase; GGT, $\gamma$-glutamyl transpeptidase; LSD, least significant difference.

† Model 2: adjusted for the factor in model 1 plus current smoking (yes or no), BMI, WHR, SBP, DBP, TAG, HDL-cholesterol, LDL-cholesterol, logUACR and leucocytes.

† MNOVA with LSD post hoc test: $P<0.05 \mathrm{v}$. first tertile.

¥ ANOVA with LSD post hoc test: $P<0.05 \mathrm{v}$. first tertile.
\& ANOVA with LSD post hoc test: $P<0.05 \mathrm{v}$. second tertile.

॥ Model 3: adjusted for factors in model 2 as well as ALT, AST and GGT. 
those not using hormone therapy ${ }^{(19)}$. However, the relationship between ferritin and IR appeared to be statistically significant when comparing the second and third tertiles of ferrtin with the lowest tertile (the first tertile) in the whole group of study participants after adjusting for age, sex, current smoking, BMI, WHR, SBP, DBP, logUACR, leucocytes, lipid profiles and liver enzymes (data not shown). The null association between ferritin and IR in the pre-menopausal women in our study may be due to the limited sample size and a relatively small proportion compared with men and post-menopausal women.

However, some studies have shown a lack of consistency in the association between circulating ferritin and IR according to sex and menopausal status ${ }^{(2-4)}$. A recent Chinese study showed that elevated serum ferritin levels were independently associated with IR regardless of $\operatorname{sex}^{(3)}$. Chen et al.'s ${ }^{(3)}$ study excluded participants with a known history of diabetes, liver disease, chronic or acute inflammatory disease or atherosclerosis, similarly as ours. It should be noted, the BMI, blood pressure and blood glucose of the female participants in our study were relatively improved in comparison with those in Chen et al.'s, which may contribute to the insignificant correlation in pre-menopausal women. The median values of HOMA-IR were markedly lower in our study compared with that in Chen et al.'s (1.5 and 1.7 in premenopausal and post-menopausal women $v$. 1.83). The fact that no association was observed for pre-menopausal women in our study may be due to the aforementioned different results. Pham et $a l^{(2)}$ reported that serum ferritin levels were positively associated with HOMA-IR in men but not in women, even among post-menopausal women in a Japanese working population aged 20-68 years old. The mean values of ferritin were significantly lower in the Japanese population, compared with those in our study $(23.4$ and $75.4 \mathrm{ng} / \mathrm{ml}$ in pre-menopausal and postmenopausal women in Japanese, respectively $v$. 94.6 and $179.8 \mathrm{ng} / \mathrm{ml}$ in ours). Ferritin measurement methods generally vary widely among measurement methods ${ }^{(20)}$. The earlier two studies used different ferritin measurement methods. If there is a positive association between ferritin and IR markers, the association would not be discernible in participants who have lower concentrations of serum ferritin. These may explain the discrepancy found in the link between ferritin levels and IR in the women. Moreover, statistical power could also explain the discrepant findings, because most of the studies describing associations had larger sample sizes for pre-menopausal women than those with no association ${ }^{(21-23)}$.

Our study provided an insight into the finding of intriguing relationships between ferritin and increased $\beta$-cell function in post-menopausal participants. However, most studies reported that serum ferritin levels were associated with decreased $\beta$-cell function $^{(24)}$. As high serum ferritin levels is a risk marker for the development of IR in post-menopausal women, enhanced insulin secretion by $\beta$ cells in response to the increasing IR is a reasonable compensatory mechanism, and subsequently, the compensatory ability possibly starts to fail, resulting in the decreased $\beta$-cell function. Similarly, in a cross-sectional study, a weak and direct association was observed between ferritin quartiles and $\beta$-cell function using HOMA- $\% \mathrm{~B}$ in normoglycaemia, which was direct in the case of pre-diabetes and inverse in $\mathrm{DM}^{(25)}$
The mechanisms for the effect of ferritin on IR remain unknown, although several explanations have been suggested. First, Fe is a major catalyst in the production of highly reactive hydroxyl radicals and excess body Fe may be directly involved in insulin signalling ${ }^{(26)}$. Second, Fe causes the peroxidation of lipids, especially free fatty acids, leading to accelerated production of free radicals; thereafter, the increased free fatty acids oxidation causes decreased glucose uptake in the muscles, which stimulates gluconeogenesis in the liver and results in increased $\mathrm{IR}^{(27)}$. Third, Fe negatively regulates adiponectin transcription via forkhead box protein O1 (FOXO1)-mediated repression. Serum ferritin level was increased and adiponectin level was decreased in type $2 \mathrm{DM}$ and in obese diabetic participants $^{(28)}$. Lower plasma adiponectin concentrations in men than women contribute to sex differences in insulin sensitivity $^{(29)}$. Finally, Fe was closely related to inflammation, which imposed a higher risk on IR. However, in our study after adjusting for leucocytes and UACR, the associations between serum ferritin and IR were still significant, which might be suggestive of other pathways of the effects of serum ferritin on IR. Another possible explanation is that Fe deposition in the liver can result in hepatic IR and increase hepatic glucose production $^{(30)}$. Furthermore, other studies have suggested a link between serum ferritin and nonalcoholic fatty liver disease, currently regarded as one of the independent risk factors for $\mathrm{IR}^{(31)}$. We did observe a positive relationship between the ferritin tertile groups and liver enzymes in male and postmenopausal participants (i.e. ALT, AST and GGT; Table 2).

IR plays an important role in the development of diabetes, hypertension, the metabolic syndrome and atherosclerotic diseases ${ }^{(32,33)}$. The incidence of IR is increasing and early management has become important to prevent patients from developing more severe diseases. Decreased Fe stores resulting from frequent blood donation or bloodletting have been associated with improved $\mathrm{IR}^{(34,35)}$, supporting the notion that serum ferritin may become a useful biomarker to predict IR. Our findings underscore the public health importance of monitoring ferritin level, and also suggested that Fe metabolism may underlie the aetiology of glucose homoeostasis.

Furthermore, the association between serum ferritin levels and DM has been broadly concerned in China. To date, several case-control studies and prospective studies have investigated the association; however, their results were inconsistent ${ }^{(36-40)}$. Possible reasons for the differences among the aforementioned studies include the study population, the length of the study and the definition of diabetes.

Our study has several strengths. Serum ferritin is considered to be the best indicator of body Fe stores, despite being shown to be affected by inflammation status. As mentioned in previous studies $^{(41)}$, ferritin can be elevated during inflammation, as well as in the case of cancer and liver disease. Serum ferritin concentration can also be increased in some conditions like obesity and non-alcoholic fatty liver disease, which are associated risk factors for $\mathrm{IR}^{(42)}$. We controlled for possible confounding in the study by several ways. All participants had no confounding co-morbidities such as CVD, anaemia, renal disease, liver diseases and chronic inflammatory diseases ${ }^{(34)}$. We restricted the study population to those without overt liver disease, 
defined as no more than two times of the upper normal limits in liver enzymes. Therefore, it provided a unique opportunity to study the association between the markers of Fe metabolism and IR in the middle-aged and elderly population. Moreover, the association remained robust even after adjustment for multiple confounding factors including inflammation controlling for leucocytes and UACR levels, and ALT, AST and GGT for liver diseases in the multivariate models, which have seldom been accounted for in the previous studies.

The present study had several limitations that should be acknowledged. First, standard measurements of IR and $\beta$-cell dysfunction (hyperinsulinaemic-euglycaemic and hyperglycaemic clamps) were not used in our study. However, HOMA-IR and HOMA-\%B are the most employed tools in epidemiological studies. Second, the cross-sectional design does not allow us to determine a causal relationship among the evaluated variables. Third, we could not adjust for dietary factors due to lack of data on dietary haem $\mathrm{Fe}$ intake. Fourth, socio-economic factors are associated with lifestyles and behavioural risk factors. However, we do not have data on the socio-economic factors and lifestyle factors including physical activity and energy intake. Notably, recent studies found that, among the behavioural risk factors considered, BMI and waist circumference may serve as a lifetime proxy for diet quality and level of physical activity of health behaviours ${ }^{(43,44)}$. We have already fully adjusted for BMI and waist circumference in the study. Fifth, our participants were middle-aged and elderly participants, and so, the results cannot be applied to younger participants. Moreover, we cannot rule out the residual confounding effects due to a failure to adjust for C-reactive protein, as serum ferritin is an acute-phase reactant and may be artificially elevated in the presence of inflammation $^{(45)}$

Fe element is an inorganic constituent needed in minute quantities but considered an essential nutrient for human health. Therein, it is worth noting that deficiency or the excessive accumulation of Fe element could be detrimental. In the context of human health, screening based on ferritin would enable clinicians to provide early interventions. The individuals with increased or decreased ferritin levels might benefit from more aggressive lifestyle modifications and dietary regimen. The supply of three macro-nutrients (carbohydrate, protein and lipid) was sufficient in mainland China ${ }^{(46)}$. However, it was a common issue that there was deficient intake of micronutrients such as $\mathrm{Fe}$ and $\mathrm{Zn}$, most of them being among women and children living in rural areas ${ }^{(46)}$. Fe supplementation and fortification can be used as short-term interventions, while dietary diversification and biofortification could be long-term interventions ${ }^{(47)}$.

In conclusion, we evaluated ferritin and glucose homoeostasis obtained from OGTT in 2518 middle-aged and elderly Chinese individuals and observed that elevated fasting serum ferritin levels were associated with surrogate measures of increased IR in both men and post-menopausal women, but not among pre-menopausal women, whereas the association with increased $\beta$-cell function was present in post-menopausal women. These findings suggest that ferritin may contribute to the development of IR. Therefore, our study added further insight into the public health importance of monitoring ferritin level.

\section{Acknowledgements}

The Shanghai Changfeng Study has also received great support from Changfeng Health Center, the Health Bureau of Putuo District and the committees of all the sub-communities of Changfeng. The contributions of all the working staffs and inhabitants are greatly acknowledged.

This work was supported by grants from the Shanghai Science and Technology Committee (STCSM) Foundation (16411954800 to X. G.), and the Three-year Action Plan on Public Health, Phase IV, Shanghai, China (grant no. 15GWZK0801 to H. L. and X. G.).

H. M., H. L., X. L., Y. H., X. J., J. G., N. Z., B. P. and X. G. conceived and designed the experiments. H. M., H. L., X. L., W. H., J. G. performed the experiments. H. M., N. Z., J. G. analysed the data. H. M., X. G. wrote the paper. All authors read and approved the final manuscript.

The authors declare that there are no conflicts of interest.

\section{References}

1. Zacharski L, Ornstein D, Woloshin S, et al. (2000) Association of age, sex, and race with body iron stores in adults: analysis of NHANES III data. Am Heart J 140, 98-104.

2. Pham NM, Nanri A, Yi S, et al. (2013) Serum ferritin is associated with markers of insulin resistance in Japanese men but not in women. Metabolism 62, 561-567.

3. Chen L, Li Y, Zhang F, et al. (2017) Association of serum ferritin levels with metabolic syndrome and insulin resistance in a Chinese population. J Diabetes Complications 31, 364-368.

4. Krisai P, Leib S, Aeschbacher S, et al. (2016) Relationships of iron metabolism with insulin resistance and glucose levels in young and healthy adults. Eur J Intern Med 32, 31-37.

5. Yeap BB, Divitini ML, Gunton JE, et al. (2015) Higher ferritin levels, but not serum iron or transferring saturation, are associated with type 2 diabetes mellitus in adult men and women free of genetic haemochromatosis. Clin Endocrinol 82, 525-532.

6. Bonfils L, Ellervik C, Friedrich N, et al. (2015) Fasting serum levels of ferritin are associated with impaired pancreatic beta cell function and decreased insulin sensitivity: a populationbased study. Diabetologia 58, 523-533.

7. Kim CH, Kim HK, Bae SJ, et al. (2011) Association of elevated serum ferritin concentration with insulin resistance and impaired glucose metabolism in Korean men and women. Metabolism 60, 414-420.

8. Zhan Y, Tang Z \& Yu J (2014) Serum ferritin, diabetes, diabetes control, and insulin resistance. Acta Diabetol 51, 991-998.

9. Harris EL, McLaren CE, Reboussin DM, et al. (2007) Serum ferritin and transferrin saturation in Asians and Pacific Islanders. Arch Intern Med 167, 722-726.

10. Gao X, Hofman A, Hu Y, et al. (2010) The Shanghai Changfeng Study: a communitybased prospective cohort study of chronic diseases among middle-aged and elderly: objectives and design. Eur J Epidemiol 25, 885-893.

11. Matthews DR, Hosker JP, Rudenski AS, et al. (1985) Homeostasis model assessment: insulin resistance and beta-cell function from fasting plasma glucose and insulin concentrations in man. Diabetologia 28, 412-419.

12. Chobanian AV, Bakris GL, Black HR, et al. (2003) Seventh report of the Joint National Committee on Prevention, Detection, Evaluation, and Treatment of High Blood Pressure. Hypertension 42, 1206-1252. 
13. American Diabetes Association (2010) Standards of medical care in diabetes - 2010. Diabetes Care 33, S11-S61.

14. Lee BK, Kim Y \& Kim YI (2011) Association of serum ferritin with metabolic syndrome and diabetes mellitus in the South Korean general population according to the Korean National Health and Nutrition Examination Survey 2008. Metabolism 60, 1416-1424

15. Jehn M, Clark JM \& Guallar E (2004) Serum ferritin and risk of the metabolic syndrome in U.S. adults. Diabetes Care 27, 2422-2428.

16. Mateo-Gallego R, Calmarza P, Jarauta E, et al. (2010) Serum ferritin is a major determinant of lipid phenotype in familial combined hyperlipidemia and familial hypertriglyceridemia. Metabolism 59, 154-158.

17. Geer EB \& Shen W (2009) Gender differences in insulin resistance, body composition, and energy balance. Gend Med 6, Suppl. 1, 60-75.

18. Doshi SB \& Agarwal A (2013) The role of oxidative stress in menopause. J Midlife Health 4, 140-146.

19. Penckofer S \& Schwertz D (2000) Improved iron status parameters may be a benefit of hormone replacement therapy. J Womens Health Gend Based Med 9, 141-151.

20. Kamei D \& Akiba T (2015) Coefficient of variation among ferritin measurement methods in hemodialysis and standard serum ferritin level. Kidney Int 88, 197.

21. Yoo KD, Ko SH, Park JE, et al. (2012) High serum ferritin levels are associated with metabolic risk factors in non-obese Korean young adults: Korean National Health and Nutrition Examination Survey (KNHANES) IV. Clin Endocrinol (Oxf) 77, 233-240.

22. Yoon JH, Linton JA, Koh SB, et al. (2012) Serum ferritin concentrations predict incidence of metabolic syndrome in rural Korean adults. Clin Chem Lab Med 50, 2057-2059.

23. Cho GJ, Shin JH, Yi KW, et al. (2011) Serum ferritin levels are associated with metabolic syndrome in postmenopausal women but not in premenopausal women. Menopause 18, 1120-1124.

24. Vari IS, Balkau B, Kettaneh A, et al. (2007) DESIR Study Group. Ferritin and transferrin are associated with metabolic syndrome abnormalities and their change over time in a general population: Data from an Epidemiological Study on the Insulin Resistance Syndrome (DESIR). Diabetes Care 30, 1795-1801.

25. Aregbesola A, Virtanen JK, Voutilainen S, et al. (2015) Serum ferritin and glucose homeostasis: change in the association by glycaemic state. Diabetes Metab Res Rev 31, 507-514.

26. Messner DJ, Rhieu BH \& Kowdley KV (2013) Iron overload causes oxidative stress and impaired insulin signaling in AML-12 hepatocytes. Dig Dis Sci 58, 1899-1908.

27. Aregbesola A, Virtanen JK, Voutilainen S, et al. (2015) Serum ferritin and glucose homeostasis: change in the association by glycaemic state. Diabetes Metab Res Rev 31, 507-514.

28. Gabrielsen JS, Gao Y, Simcox JA, et al. (2012) Adipocyte iron regulates adiponectin and insulin sensitivity. J Clin Invest 122, 3529-3540.

29. Nishizawa H, Shimomura H, Kishida K, et al. (2002) Androgens decrease plasma adiponectin, an insulinsensitizing adipocyte-derived protein. Diabetes 51, 2734-2741.

30. Forouhi NG, Harding AH, Allison M, et al. (2007) Elevated serum ferritin levels predict new-onset type 2 diabetes: results from the EPIC-Norfolk prospective study. Diabetologia 50, 949-956.
31. Kowdley KV, Belt P, Wilson LA, et al. (2012) Serum ferritin is an independent predictor of histologic severity and advanced fibrosis in patients with nonalcoholic fatty liver disease. Hepatology 55, 77-85.

32. Cho MR, Park JK, Choi WJ, et al. (2017) Serum ferritin level is positively associated with insulin resistance and metabolic syndrome in postmenopausal women: a nationwide population-based study. Maturitas 103, 3-7.

33. Han LL, Wang YX, Li J, et al. (2014) Gender differences in associations of serum ferritin and diabetes, metabolic syndrome, and obesity in the China Health and Nutrition Survey. Mol Nutr Food Res 58, 2189-2195.

34. Houschyar KS, Ludtke R, Dobos GJ, et al. (2012) Effects of phlebotomy-induced reduction of body iron stores on metabolic syndrome: results from a randomized clinical trial. $B M C$ Med 10, 54 .

35. Fernández-Real JM, López-Bermejo A \& Ricart W (2005) Iron stores, blood donation, and insulin sensitivity and secretion. Clin Chem 51, 1201-1205.

36. Guo X, Zhou D, An P, et al. (2013) Associations between serum hepcidin, ferritin and $\mathrm{Hb}$ concentrations and type 2 diabetes risks in a Han Chinese population. Br J Nutr 110, 2180-2185.

37. Zhou F, Zhao Z, Tian L, et al. (2016) Association of serum ferritin level with risk of incident abnormal glucose metabolism in southwestern China: a prospective cohort study. Biol Trace Elem Res 169, 27-33.

38. Gao S, Zhao D, Qi Y, et al. (2017) The association between serum ferritin levels and the risk of new-onset type 2 diabetes mellitus: a 10-year follow-up of the Chinese Multi-Provincial Cohort Study. Diabetes Res Clin Pract 130, 154-162.

39. Sun L, Zong G, Pan A, et al. (2013) Elevated plasma ferritin is associated with increased incidence of type 2 diabetes in middleaged and elderly Chinese adults. J Nutr 143, 1459-1465.

40. Chen L, Li Y, Zhang F, et al. (2018) Elevated serum ferritin concentration is associated with incident type 2 diabetes mellitus in a Chinese population: a prospective cohort study. Diabetes Res Clin Pract 139, 155-162.

41. Eftekhari MH, Mozaffari-Khosravi H, Shidfar F, et al. (2013) Relation between body iron status and cardiovascular risk factors in patients with cardiovascular disease. Int J Prev Med 4, 911-916.

42. Kitade H, Chen G, Ni Y, et al. (2017) Nonalcoholic fatty liver disease and insulin resistance: new insights and potential new treatments. Nutrients $\mathbf{9}, 4$.

43. Yang MH, Hall SA, Piccolo RS, et al. (2015) Do behavioral risk factors for prediabetes and insulin resistance differ across the socioeconomic gradient? Results from a community-based epidemiologic survey. Int J Endocrinol 2015, 806257.

44. Stringhini S, Sabia S, Shipley M, et al. (2010) Association of socioeconomic position with health behaviors and mortality. JAMA 303, 1159-1166.

45. Gabay C \& Kushner I (1999) Acute-phase proteins and other systemic responses to inflammation. N Engl J Med 340, 448-454.

46. Guo QY, Zhao LY, He YN, et al. (2017) Survey on dietary nutrients intake of Chinese residents between 2010 and 2012. Zhonghua Yu Fang Yi Xue Za Zbi 51, 519-522.

47. Ma G, Jin Y, Li Y, et al. (2008) Iron and zinc deficiencies in China: what is a feasible and cost-effective strategy? Public Health Nutr 6, 632-638. 